\title{
A Systematic Review On Correlates of Risk of TB Disease In Children And Adults
}

P Siddhi ( $\sim$ p.siddhi@nhs.net )

Walsall Manor Hospital

R Raveendranath

Medical University of Lodz

A Chinnaswamy

Aston University

P Pulgari

Sandwell and West Birmingham Hospitals

R Song

University of Oxford

Steven Welch

University Hospitals Birmingham

\section{Research Article}

Keywords: Correlates of risk (COR), TB disease, systematic review

Posted Date: February 3rd, 2022

DOI: https://doi.org/10.21203/rs.3.rs-1261861/v1

License: (c) (i) This work is licensed under a Creative Commons Attribution 4.0 International License.

Read Full License 


\section{Abstract}

Background: Tuberculosis (TB) remains one of the leading causes of death in the world. Targeted treatment to prevent progression from TB exposure and infection to disease is a key element of WHO End-TB strategy. A systematic review to identify and develop correlates of risk (COR) of TB disease is timely.

Method: EMBASE, MEDLINE, PUBMED were searched using relevant keywords and MeSH terms published between 2000-2020 on COR of TB disease in children and adults. Preferred Reporting Items for Systematic reviews and Meta-analysis (PRISMA) framework was used for structuring and reporting of outcomes. Risk of bias was assessed using Quality Assessment of Diagnostic Accuracy Studies tool-2 (QUADAS-2).

Results: 4105 studies were identified. Following eligibility screening, 27 studies were quality assessed. Risk of bias was high in all studies. Broad variations in COR type, study population, methodology and result reporting were observed. Tuberculin skin test (TST) and interferon gamma release essays (IGRA) are poor COR. Transcriptomic signatures although promising require validation studies to assess wider applicability. Performance consistency of other CORs- cell marker, cytokines and metabolites are much needed.

Conclusion: This review identifies the need for a standardized approach to identify a universally applicable COR signature to achieve the WHO END-TB targets.

\section{Background}

Tuberculosis (TB) is one of the top 10 causes of death globally. In 2019 , there were an estimated 10 million new TB cases including 1.2 million children worldwide ${ }^{1}$. Of the one-fourth of the world's population who are infected only $5-10 \%$ evolve into active disease (TB disease) during their lifetime.

The majority of TB disease in the world is caused by Mycobacterium tuberculosis (M.tuberculosis). The susceptibility of the host (immune status), the infectiousness of the transmitter (number of tubercle bacilli that are expelled), the environmental factors and the duration of exposure all influence the probability of transmission ${ }^{2}$.

TB affects all age groups and once infected progression to disease is greater in the first two years after being infected ${ }^{3}$. Children ( $<5$-years of age), individuals with HIV and those immune-suppressed are at increased risk of progression to TB disease ${ }^{4}$. Malnutrition and vitamin D deficiency have been associated with risk of TB disease progression (reference). Genetic deficiencies related to IFNY and IL-12 are linked to an increased susceptibility to less virulent mycobacteria that causes severe disseminated and/ or recurrent infections termed as mendelian susceptibility to mycobacterial diseases (MSMD) ${ }^{5}$. 
Current available TB screening tests, tuberculin skin test (TST) and interferon gamma release assays (IGRA) identify host immune responses suggestive of either an exposure to TB or evidence of a chronic non-progressive state. Uncertainty in screening is common, particularly when cross-reaction with other atypical mycobacterium occurs (TST) or an indeterminate IGRA result is encountered.

Bacteriological confirmation (culture or PCR) is the gold standard for TB disease diagnosis. Mycobacterial Growth Indicator Tube (MGIT-BACTEC) a liquid culture is a sensitive method to isolate mycobacterium in comparison to the solid culture method (Lowenstein- Jenson medium) ${ }^{6}$. Xpert MTB/RIF (Nucleic Acid Amplification test) by Cepheid, was endorsed by WHO in 2011 as the next generation assay to diagnose TB disease. An advantage over culture is that it can detect Mycobacterium $T B$ complex and resistance to rifampicin in less than two hours. Xpert MTB/RIF is reported to have a sensitivity of $64 \%$ in sputum samples and a specificity of $98 \%$ in smear positive samples ${ }^{7}$. The nextgeneration assay, Xpert MTB/RIF Ultra incorporates a PCR amplification technique and two additional molecular targets for Mycobacterium tuberculosis. In adults, Xpert MTB/RIF Ultra studies demonstrated an improved sensitivity both in smear positive and smear negative specimen ${ }^{8}$. Two accuracy studies in children conducted on cryo-preserved samples showed an increment in sensitivity by $11 \%$, and a specificity of $97 \%$.

Considering a fourth of the world's population are latently infected, offering prophylactic treatment for such huge numbers is impractical. A consensus meeting report (2017) by WHO and the Foundation for Innovative New Diagnostics (FIND) on the development of a Target Product Profile (TPP) for a test predicting the progression to TB disease outlined operational and performance characteristics for predictive tests and recommendations for standardization of future studies. A minimum sensitivity and specificity for a TB predictive test of $\geq 75 \%$ and specificity of $\geq 90 \%$ was set ${ }^{10}$.

To date a few systematic reviews on biomarkers with TB disease diagnostic potential have been published $^{11} 12$. One systematic review /metanalysis on patient level pooled transcriptomic signatures for diagnosis of incipient TB state was published by Gupta et al. (2020). This evaluated only transcriptomic signatures as TB disease predictors on adult blood samples overlooking other potential serological markers.

In this systematic review, we aim to evaluate outcomes of studies on non- sputum-based COR of TB disease in children and adults, compare the results against the TPP targets for a TB prognostic test and identify the knowledge gaps for future exploratory studies.

\section{Method}

A systematic review on COR TB disease in children and adults published between January $1 \mathrm{st}, 2000$ to May 25th, 2020 was conducted. EMBASE, MEDLINE, PUBMED were searched for relevant publications. In addition, key journals were searched. English language, year of publication and age groups [infants- (0-1 
year), children (1-10 years), adolescents (10-19 years) and adults (19-65 years)] were used as filters. For each database, the search terms were transposed as appropriate.

\section{Identification of relevant studies}

Table-1 illustrates the keywords that were transcribed appropriately in each database searched.

\section{Eligibility criteria}

To ensure the selection of relevant studies for this review, the study selection was guided by the eligibility criteria as specified under the inclusion/exclusion criteria.

\section{Inclusion criteria}

We included studies that met the following criteria:

1. Study question- studies that addressed the review question i.e. COR of TB disease progression were included.

2. Study type- Case-control, longitudinal cohort studies that utilized new study samples/subjects and studies that included prior recruited study samples/subjects were included.

3. Study subjects- studies with human subjects were included. And ages between 0-65 years were included.

4. COR-studies that included COR derived from host immune response to TB mycobacterium were included.

\section{Exclusion criteria}

Studies on correlates of treatment (COT) and correlates of diagnosis (COD) of latent and TB disease were excluded. Cross-sectional studies, conference papers and review articles were excluded. Studies on animal subjects were excluded.

\section{Study Selection}

A structured preparation and reporting of systematic review in line with Preferred Reporting Items for Systematic Reviews and Meta-Analysis (PRISMA) guidelines was performed ${ }^{13}$.

The data extraction criteria were developed electronically using google forms. The Table-2 shows the data extraction form that was used.

Titles of the studies were screened by one of the authors (PS) and exclusions were made based on- target condition (non-LTBI /TB infection and non-TB disease were excluded), and publication type (review articles, meta-analysis and conference abstracts were excluded). Abstracts and full-text studies were reviewed for eligibility by PS and RR independently. Inter-reviewer disagreements were resolved through consensus decision process overviewed by SW and RS. Data extraction was completed by PS utilizing google forms. For the purpose of standardization and consistency, one signalling question was selected 
in each domain of the QUADAS-2 framework and this process was completed by PS. In domain 1 (patient selection), signalling question on consecutive or convenience sampling was considered relevant to assess the sampling strategy. Under domain 2, signalling question on the conduct and interpretation of the index test (i.e. blinding) was chosen to be relevant. "Index test" indicates the biomarker test of prognostication. Under domain 3 , signalling question on conduct and interpretation of the reference standard was considered relevant. "Reference standard" indicates either a culture proven or an agreed composite standard to classify latent TB and/ or TB disease states was used. Under Domain 4, signalling question on patient flow was regarded relevant. The terms "Yes", "No" and "Unclear" were stated for each study for each domain.

If all responses in all four domains were "yes", then the risk of bias was considered "low" and "low applicability concerns", however if any of the responses were "no" or "unclear" then the risk of bias was considered "high" and hence "high applicability concerns".

\section{Results}

4105 studies were identified from the search. A further five studies were identified from references of review articles. Twenty-seven duplicates were eliminated. Titles of the resulting 4083 studies were screened, based on:

- Target condition (non-LTBI/TB studies) - 3699 were excluded

- Review/meta-analysis/conference abstracts - 73 were excluded

3772 studies were eliminated in the screening phase. The resulting 311 (abstract and full-text) studies were assessed for eligibility. 284 studies were excluded based on the eligibility criteria. Finally, twentyseven studies qualified for quality assessment (Figure-1).

Quality of studies (Figure:2)

1)Patient selection - (Signalling question- Was a consecutive or convenience sampling of patients used?) Fifteen studies used consecutive sampling ${ }^{1415161718192021} 222324252627$. Twelve studies used convenience sampling 282930 .

2)Index test - (Signalling question- was the conduct and interpretation of the index test blinded?) Overall, eight studies clearly stated and implemented the process of blinding 303132333435 . No clarity on the process of blinding in either the conduct or interpretation in five studies 14172832 . Fourteen studies had no documented process of blinding in their conduct and interpretation.

3)Reference standard: (Signalling question- the reference standard likely to correctly classify the target condition (i.e. LTBI/ TB infection and TB disease). Twelve studies specified use of microbiological culture or a composite standard as a reference 19202228303132333435363738 . And three studies had no clear 
definition of reference standard ${ }^{161831}$. Twelve studies lacked clarity in defining reference standard to classify target condition.

4)Patient flow and timing- (Signalling question- were all participants included in the analysis?) Eight studies had a clear account on all the participants included in the analysis of the study 14192429333536 39. In five studies exclusion of participants was unclear 1825263234 . Fourteen studies did not account for all participants and no explanations were provided.

Hence a "high risk" of bias was observed in the studies and hence a "high applicability" concerns. (Figure:3)

\section{Characteristics of studies Year of publication}

All studies included were published between 2007 and 2020. Nineteen (70\%) studies were published in the last five-years (2016-2020). Thirteen (48\%) were conducted in sub-Saharan Africa, seven in Europe, six in Asia and one in South America. Based on the WHO classification of TB endemicity- twenty (74\%) studies were conducted in high TB incidence countries (Incidence rate $>40$ per 100,000 population) and seven (26\%) studies were conducted in low TB incidence countries (incidence rate $<40$ per 100,000 population).

\section{Study population}

Mean sample size in the twenty-seven studies was 745(n). Mean number of study subjects who developed TB disease during the study period was 42 (n). Nineteen (70\%) studies included adult, children and adolescent groups. Five studies included the Adolescent Cohort Study (ACS) with 6363 healthy adolescents aged between 12-18years in South Africa 3033353638 . Four studies included the Grand Challenges 6-74 (GC6-74) cohort with 4462 healthy household contacts of 1098 index TB cases in Africa ${ }^{33}$.Median duration of studies was two years (IQR-1.5 -5 years). Twenty $(74 \%)$ studies evaluated $>1$ COR for TB disease (Table-1).

\section{A. Analysis based on COR type i)Tuberculin skin test (TST) based}

Five cohort studies reported TST as a COR of TB disease 2021232731 . The risk of bias was high in all studies. Four were conducted in low endemic countries and one in a high endemic country. Despite a large sample size, the proportion developing TB disease was low (range 0.9-2.5\%). As a COR for TB disease, a pooled sensitivity of $94 \%$ and specificity of $44 \%$ was calculated.

\section{ii)IGRA based}


Ten cohort studies reported on IGRA 17192021232425293140 . The risk of bias was high in all studies. Seven were conducted in low endemic countries. Despite a large sample size, the proportion developing disease was low (Range-0 - 3.1\%). As a COR for TB disease, a pooled sensitivity of $90 \%$ and specificity of $86 \%$ was calculated. PPV was reported to be $<4 \%$ in four studies.

\section{iii)Cytokine based}

Nine studies on cytokines were identified 151617182628303738 . Five were case-control and four were cohort studies. Eight were conducted in high TB endemic regions. A high risk of bias was noted in these studies. Three studies had large sample sizes, however the percentage who progressed to have TB disease was $<1 \%$. Four studies were on IL-10 based on children and adults and demonstrated no consistent correlation to TB disease progression 40411638 . Complement factors were studied in two studies. Both were South African based case-control studies (validation sets) on adolescent and adult patient groups that demonstrated good correlation to TB disease progression ${ }^{38} 29$.

\section{iv)Cell marker based}

All five studies were conducted in high TB endemic regions 2728373839 . Two case-control and three cohort studies were identified. The risk of bias was high in all studies. Large sample sizes were noted in these studies and the proportion who progressed to have TB disease at the end of the study period ranged between 0.9\%-35\%. Monocyte levels were reported in four studies. An aHR of 6.25 (95\% Cl-1.6323.95) and 1.17 (95\% Cl- 1.01-1.34) was reported in infants and children ${ }^{27}$. Identification of HLA DR+ CD4+ cells in infants exposed to TB (part of the MVA85A vaccine trial) increased the risk of developing TB disease (OR -1.12 (95\% Cl-1.05-1.19) ${ }^{28}$.

\section{v)Antibody based}

Two antibodies-based studies in adults were identified. Both studies had high risk of bias ${ }^{1722}$. The proportion who developed TB disease at the end of the study period were negligible. Hence outcomes reported were not significant.

\section{vi)Transcriptional signature based}

Eight transcriptional signature studies were conducted in high TB endemic countries 1429323335363738 . All were case-control and six had validation sets included. The studies included 16-gene, RISK-4, 3-gene, PREDICT-29 and c-miRNA signatures and predominantly included adolescent and adult cohort from South Africa. Two studies reported on the 16-gene signature ${ }^{33} 36$. A large sample size was observed in all studies, and the proportion who developed TB disease at the end of the study period ranged between 0.9\%-29\%. 16 gene, 3-gene, PREDICT-29 reported sensitivities and specificities of $66 \%, 86 \%, 74 \%$ and $81 \%$, $84 \%, 85 \%$ respectively in the test sets. However, in the validation sets, 16-gene and PREDICT-29 signatures reported sensitivities and specificities of $54 \%, 56 \%$ and $83 \%, 76 \%$ respectively. RISK 4 signature and the cmiRNA studies reported AUC of 0.7 (95\% Cl-0.58-0.82) and 0.67 (95Cl-0.57-0.77) respectively.

\section{vii) Metabolite based}


A case-control study on 4462 adolescents and adults in the sub-Saharan African region, reported a sensitivity-50\%, specificity- $75 \%$, AUC- 0.68 (95\% Cl-0.64-0.73) on TB-Healthy signature ${ }^{34}$. The proportion of those who developed TB disease was 3\%.

\section{B. Performance of $\mathrm{COR}$ in high-risk groups}

Twelve studies included high risk groups- ten studies on infants and children, one on HIV infected adults, one on HIV exposed and un-infected infants, one on malnourished adolescents and adults and one on Rheumatoid Arthritis (RA) adults. A lack of correlation (not statistically significant) of IFN against Heparin binding haemagglutinin antigen (HBHA) in HIV +ve adults was observed. A higher neopterin levels in RA patients who developed TB was observed (median $-24.5 \mathrm{pg} / \mathrm{ml}$ and $23053 \mathrm{pg} / \mathrm{ml}$ respectively) and non-RA TB patients $(12.2 \mathrm{pg} / \mathrm{ml}$ and $9633 \mathrm{pg} / \mathrm{ml}$ respectively) compared with QFT-Gold converters without TB $(3 \mathrm{pg} / \mathrm{ml}$ and $2720 \mathrm{pg} / \mathrm{ml}$ respectively both $\mathrm{p}<0.01)$. Alpha-2 macroglobulin, sero-transferrin, haptoglobin levels in malnourished endemic population demonstrated a two-fold increase in levels, however the number/s followed up were negligible.

\section{COR and WHO TPP targets}

WHO and FIND set an optimal TPP for a TB predictive test with a sensitivity and specificity of $\geq 90 \%$ or a minimum of $\geq 75 \%$ (within 2-years of exposure) and a high PPV with low NNT. Outcomes of five included studies could be compared against the WHO TPP targets (cytokine signature-3PR, TRM- ${ }^{30}$,

transcriptomic signatures- 16 -gene ${ }^{33}, 3$-gene ${ }^{36}$, PREDICT-29 ${ }^{14}$ and metabolomic signature-TB-healthy ${ }^{34}$ ) The performance of cytokine signatures as COR were comparable in the test sets but fell short in the validation sets. Similarly, the performance of 16-gene signature and PREDICT29 signature fell short of comparison in the validation sets. The 3-gene transcriptomic signature showed consistency in its performance in the nested cohort (validation set) and was comparable to the targets set for a TB predictive test. (Figure:4)

\section{Discussion}

We conducted a systematic review on blood-based, host-derived COR for TB disease in children and adults in accordance with PRISMA framework. We intended to analyze the outcomes reported by studies on TB COR and compare their performance against the WHO/FIND TPP targets. TST and IGRA are poor COR of TB disease progression. A meta-analysis reported a PPV of $2.4 \%$ for TST and $6.8 \%$ for IGRA, implying the number needed to treat (NNT) to prevent one case of TB disease to be as high as 67.3 for TST and 37.3 for IGRA ${ }^{25}$. Among the cytokine studies, IL-10 (anti-inflammatory) was analyzed in five studies that demonstrated poor co-relation to TB disease progression ${ }^{1718293738}$. Complement components ${ }^{3038}$ and M:L ratio ${ }^{272939}$ demonstrated a good correlation to TB progression, however larger prospective studies in diverse populations are required. Activated T-cell as a COR can be confounded by several factors that include environmental triggers and childhood immunization and hence this is a poor predictor in children ${ }^{28}$. Studies on transcriptomic signatures have demonstrated the most encouraging results to date ${ }^{14323335363738}$. However, their predictive performance when validated in geographically 
diverse cohorts reported inconsistencies. In addition, their predictive performance improved closer towards TB disease diagnosis which reduces the usefulness for early detection of incident TB for targeted preventive TB treatment. A meta-analysis on eight transcriptomic signatures reported comparable results (at pre-test probability of $2 \%$ ), PPV between $6.8-9.4 \%$ over 24 months and $11.2-14.4 \%$ over 3-months before TB disease diagnosis ${ }^{41}$. The recent CORTIS trial, a randomised open label study that tested the prognostic accuracy of RISK-11 signature (derived from Zak-16 signature) and efficacy of targeted TB preventive treatment reported that the signature performed well as a COR, however, the targeted preventive TB treatment in comparison to signature positives and negatives showed poor efficacy in preventing TB disease during the 15 -month follow-up ${ }^{42}$. Though this study incorporated diverse population in South Africa, it raises a few questions on the accuracy of the signature as a predictor of TB disease, the targeted preventive TB treatment approach and tackling the distinct complexities in high and low TB endemic regions.

This review has some potential limitations. The restricted search terminologies might have resulted in a narrow search. Only pulmonary TB studies were included in the search terminology hence that excluded studies on extra-pulmonary TB. All blood-based biomarkers were included in this review, making this review very broad. However, this review consolidates information on most potential COR studied till date. A narrative analysis was entailed due to the heterogenous nature of the studies which highlights the need for a standardized reporting for future studies in this field. A large number of studies incorporated highrisk groups, however the outcomes cannot be extrapolated into clinical practice due to the relatively small sample sizes and even smaller proportion of those evolving into TB disease. This highlights the practical challenges in conducting large longitudinal observational studies.

\section{Conclusions}

A worldwide accelerated effort to derive at a blood-based, host-derived COR for TB disease has been observed in the last two decades. TB disease progression is marked by a cascade of events and the studies on cell markers, metabolomic and transcriptomic signatures identified in this review have shown good potential as COR. As future prospects, validation studies on combination of these COR (as a signature) would be ideal.

\section{Declarations}

\section{Conflicting interest}

The authors declare that they have no potential conflict of interest regarding the submitted manuscript.

\section{Funding statement}

This research received no specific grant from any funding agency in the public, commercial, or not-forprofit sectors. 
Ethical approval and consent to participate

Not applicable.

\section{Consent for publication}

All authors have read the final manuscript and provided their consent for publication.

\section{Author's Contribution}

P.S, S.W and R.S envisaged the idea for the review and its purpose. P.S and P.P conducted the systematic literature search. P.S and R.R screened the studies as per PRISMA framework. P.S assessed the quality of the studies based on the QUADAS II framework overviewed by S.W and R.S. P.S and A.C wrote the manuscript text and prepared all figures and tables. All authors reviewed the manuscript

\section{Acknowledgements}

We would like to thank Professor Andrew Pollard for taking time and reviewing this paper.

\section{Availability of data and materials}

The authors confirm that the data supporting the findings of this study are available in the supplementary file titled Studies and outcomes.

\section{References}

1. WHO. Tuberculosis [Internet]. World Health Organization; 2020. (WHO). Available from: https://www.who.int/news-room/fact-sheets/detail/tuberculosis.

2. Grover S, Hasnain SE, Ehtesham NZ. Mycobacterium Tuberculosis: Molecular Infection Biology, Pathogenesis, Diagnostics and New Interventions [Internet]. Singapore: Springer Singapore; 2019. Available from: http://link.springer.com/10.1007/978-981-32-9413-4

3. Behr MA, Edelstein PH, Ramakrishnan L. Revisiting the timetable of tuberculosis. 2018 Aug 23;362:k2738. Available from: http://dx.doi.org/10.1136/bmj.k2738

4. WHO. Tuberculosis [Internet]. World Health Organization; 2020. (WHO). Available from: https://www.who.int/news-room/fact-sheets/detail/tuberculosis

5. Bustamante J, Boisson-Dupuis S, Abel L, Casanova J-L. Mendelian susceptibility to mycobacterial disease: Genetic, immunological, and clinical features of inborn errors of IFN- $\gamma$ immunity. 2014 Dec;26(6):454-70. Available from: https://search.datacite.org/works/10.1016/j.smim.2014.09.008

6. Brent AJ, Mugo D, Musyimi R, Mutiso A, Morpeth SC, Levin M, et al. Bacteriological diagnosis of childhood TB: a prospective observational study. 2017 Sep 18;7(1):11808-9. Available from: https://www.ncbi.nlm.nih.gov/pubmed/28924198 
7. Detjen AK, DiNardo AR, Leyden J, Steingart KR, Menzies D, Schiller I, et al. Xpert MTB/RIF assay for the diagnosis of pulmonary tuberculosis in children: a systematic review and meta-analysis. 2015 Jun;3(6):451-61. Available from: https://search.datacite.org/works/10.1016/s2213-2600(15)000958

8. Donovan J, Thu DDA, Phu NH, Dung VTM, Quang TP, Nghia HDT, et al. Xpert MTB/RIF Ultra versus Xpert MTB/RIF for the diagnosis of tuberculous meningitis: a prospective, randomised, diagnostic accuracy study. 2020 Mar;20(3):299-307. Available from: http://dx.doi.org/10.1016/S14733099(19)30649-8.

9. Atherton RR, Cresswell FV, Ellis J, Kitaka SB, Boulware DR. Xpert MTB/RIF Ultra for Tuberculosis Testing in Children: A Mini-Review and Commentary. 2019 Feb 1;7. Available from: https://explore.openaire.eu/search/publication?articleld=od 267:d1122b750442b058954f4e8c7fc4d878.

10. WHO. Consensus meeting report: development of a target product profile (TPP) and a framework for evaluation for a test for predicting progression from tuberculosis infection to active disease [Internet]. Geneva: World Health Organization; 2017. Available from: https://apps.who.int/iris/handle/10665/259176.

11. MacLean E, Broger T, Yerlikaya S, Fernandez-Carballo BL, Pai M, Denkinger CM. A systematic review of biomarkers to detect active tuberculosis. 2019 Feb 25;4(5):748-58. Available from: https://search.datacite.org/works/10.1038/s41564-019-0380-2

12. Togun TO, MacLean E, Kampmann B, Pai M. Biomarkers for diagnosis of childhood tuberculosis: A systematic review. 2018 Sep 13;13(9):e0204029. Available from: https://search.datacite.org/works/10.1371/journal.pone.0204029

13. Moher D, Liberati A, Tetzlaff J, Altman DG. Reprint-Preferred Reporting Items for Systematic Reviews and Meta-Analyses: The PRISMA Statement. 2009 Sep 1;89(9):873-80. Available from: https://search.datacite.org/works/10.1093/ptj/89.9.873

14. Leong S, Zhao Y, Ribeiro-Rodrigues R, Jones-López EC, Acuña-Villaorduña C, Rodrigues PM, et al. Cross-validation of existing signatures and derivation of a novel 29-gene transcriptomic signature predictive of progression to TB in a Brazilian cohort of household contacts of pulmonary TB. 2020 Jan; 120:101898. Available from: http://dx.doi.org/10.1016/j.tube.2020.101898

15. Bapat PR, Satav AR, Husain AA, Shekhawat SD, Kawle AP, Chu JJ, et al. Differential Levels of Alpha2-Macroglobulin, Haptoglobin and Sero-Transferrin as Adjunct Markers for TB Diagnosis and Disease Progression in the Malnourished Tribal Population of Melghat, India. 2015 Aug 4;10(8): e0133928. Available from: https://search.datacite.org/works/10.1371/journal.pone.0133928

16. Hussain R, Talat N, Ansari A, Shahid F, Hasan Z, Dawood G. Endogenously Activated Interleukin-4 Differentiates Disease Progressors and Non-Progressors in Tuberculosis Susceptible Families: A 2Year Biomarkers Follow-Up Study. 2011 Oct;31(5):913-23. Available from: https://www.ncbi.nlm.nih.gov/pubmed/21755390 
17. Talat N, Shahid F, Dawood G, Hussain R. Dynamic Changes in Biomarker Profiles Associated with Clinical and Subclinical Tuberculosis in a High Transmission Setting: A Four-Year Follow-Up Study. 2009 Jun;69(6):537-46. Available from: https://onlinelibrary.wiley.com/doi/abs/10.1111/j.13653083.2009.02250.x

18. Hussain R, Talat N, Shahid F, Dawood G. Longitudinal Tracking of Cytokines after Acute Exposure to Tuberculosis: Association of Distinct Cytokine Patterns with Protection and Disease Development. 2007 Dec 1;14(12):1578-86. Available from: http://cvi.asm.org/content/14/12/1578. abstract

19. Delogu G, Vanini V, Cuzzi G, Chiacchio T, De Maio F, Battah B, et al. Lack of Response to HBHA in HIVInfected Patients with Latent Tuberculosis Infection. 2016 Dec;84(6):344-52. Available from: https://onlinelibrary.wiley.com/doi/abs/10.1111/sji.12493

20. Kik SV, Franken WPJ, Mensen M, Cobelens FGJ, Kamphorst M, Arend SM, et al. Predictive value for progression to tuberculosis by IGRA and TST in immigrant contacts. 2010;35(6):1346-53. Available from: https://www.narcis.nl/publication/RecordID/oai:pure.amc.nl:publications\%2F266bb5c8-4782410c-b5f9-2e0dc98afedc.

21. Abubakar I, Drobniewski F, Southern J, Sitch AJ, Jackson C, Lipman M, et al. Prognostic value of interferon-y release assays and tuberculin skin test in predicting the development of active tuberculosis (UK PREDICT TB): a prospective cohort study. 2018 Jan 1;18(10):1077-87. Available from: https://explore.openaire.eu/search/publication? articleld=od267::76e61c599a1a3b8691077f65110ded02.

22. Maekura R, Kitada S, Osada-Oka M, Tateishi Y, Ozeki Y, Fujicawa T, et al. Serum antibody profiles in individuals with latent Mycobacterium tuberculosis infection. 2019 Mar;63(3-4):130-8. Available from: https://onlinelibrary.wiley.com/doi/abs/10.1111/1348-0421.12674

23. Altet N, Dominguez J, Souza-Galvão M-L de, Jiménez-Fuentes MÁ, Milà C, Solsona J, et al. Predicting the Development of Tuberculosis with the Tuberculin Skin Test and QuantiFERON Testing. 2015 May;12(5):680-8. Available from: https://www.ncbi.nlm.nih.gov/pubmed/25699406.

24. M Sascha Wilk, S Bolette, A Else Marie, D Lars Jorge, H Soren Tetens, K Anders., et al. Host immunity to Mycobacterium tuberculosis and risk of tuberculosis: A longitudinal study among Greenlanders. 2016;34(48):5975-83. Available from: https://www.clinicalkey.es/playcontent/1-s2.0S0264410X16308726.

25. Diel R, Loddenkemper R, Meywald-Walter K, Niemann S, Nienhaus A. Predictive Value of a Whole Blood IFN-y Assay for the Development of Active Tuberculosis Disease after Recent Infection with Mycobacterium tuberculosis. 2008 May 15;177(10):1164-70. Available from: https://search.datacite.org/works/10.1164/rccm.200711-1613oc

26. Chen D-Y, Li J-P, Chen Y-M, Liao T-L, Chen H-H, Hsieh C-W, et al. Elevated Neopterin Levels Are Associated with Increased Tuberculosis Risk in Rheumatoid Arthritis Patients with QuantiFERON Conversion during Biologic Therapy. 2016;11(11):e0166301. Available from: https://www.ncbi.nlm.nih.gov/pubmed/27861525 
27. Rakotosamimanana N, Richard V, Raharimanga V, Gicquel B, Doherty TM, Zumla A, et al. Biomarkers for risk of developing active tuberculosis in contacts of TB patients: a prospective cohort study. 2015 Oct;46(4):1095-103. Available from: https://search.datacite.org/works/10.1183/13993003.002632015

28. Fletcher HA, Snowden MA, Landry B, Rida W, Satti I, Harris SA, et al. T-cell activation is an immune correlate of risk in BCG vaccinated infants. 2016 Apr 12;7(1):11290. Available from: https://search.datacite.org/works/10.1038/ncomms11290

29. Fletcher HA, Filali-Mouhim A, Nemes E, Hawkridge A, Keyser A, Njikan S, et al. Human newborn bacille Calmette-Guérin vaccination and risk of tuberculosis disease: a case-control study. 2016 May 16;14(1):76. Available from: https://search.datacite.org/works/10.1186/s12916-016-0617-3

30. Penn-Nicholson A, Hraha T, Thompson EG, Sterling D, Mbandi SK, Wall KM, et al. Discovery and validation of a prognostic proteomic signature for tuberculosis progression: A prospective cohort study. 2019 Apr 16;16(4):e1002781. Available from: https://search.datacite.org/works/10.1371/journal.pmed.1002781

31. Bakir M, Millington KA, Soysal A, Deeks JJ, Efee S, Aslan Y, et al. Prognostic Value of a T-Cell-Based Interferon-Gamma Biomarker in Child Tuberculosis Contacts [Internet]. 2008. p. 777-87. (Annals of internal medicine; vol. 149). Available from: https://explore.openaire.eu/search/publication? articleld=od.267::5726805d0c59792616a3d6bd9dcea8c0.

32. Duffy FJ, Thompson E, Downing K, Suliman S, Mayanja-Kizza H, Boom WH, et al. A Serum Circulating miRNA Signature for Short-Term Risk of Progression to Active Tuberculosis Among Household Contacts. 2018;9:661. Available from: https://www.ncbi.nlm.nih.gov/pubmed/29706954.

33. Zak DE, Penn-Nicholson A, Scriba TJ, Thompson E, Suliman S, Amon LM, et al. A blood RNA signature for tuberculosis disease risk: a prospective cohort study. 2016 Jun;387(10035):2312-22. Available from: https://search.datacite.org/works/10.1016/s0140-6736(15)01316-1.

34. Weiner J, Maertzdorf J, Sutherland JS, Duffy FJ, Thompson E, Suliman S, et al. Metabolite changes in blood predict the onset of tuberculosis. 2018 Dec 6;9(1):5208-12. Available from: https://search.datacite.org/works/10.1038/s41467-018-07635-7

35. Suliman S, Thompson EG, Sutherland J, Weiner J, Ota MOC, Shankar S, et al. Four-Gene Pan-African Blood Signature Predicts Progression to Tuberculosis. 2018 May;197(9):1198-208. Available from: https://search.datacite.org/works/10.1164/rccm.201711-2340oc.

36. Warsinske HC, Rao AM, Moreira FMF, Santos PCP, Liu AB, Scott M, et al. Assessment of Validity of a Blood-Based 3-Gene Signature Score for Progression and Diagnosis of Tuberculosis, Disease Severity, and Treatment Response. 2018 Oct 5;1(6):e183779. Available from: http://dx.doi.org/10.1001/jamanetworkopen.2018.3779.

37. Sutherland JS, Hill PC, Adetifa IM, de Jong BC, Donkor S, Joosten SA, et al. Identification of Probable Early-Onset Biomarkers for Tuberculosis Disease Progression. 2011;6(9):e25230. Available from: https://www.ncbi.nlm.nih.gov/pubmed/21966464 
38. Scriba TJ, Penn-Nicholson A, Shankar S, Hraha T, Thompson EG, Sterling D, et al. Sequential inflammatory processes define human progression from $\mathrm{M}$. tuberculosis infection to tuberculosis disease. 2017 Nov 1;13(11). Available from: https://explore.openaire.eu/search/publication? articleld=od267::5837b114ee8f37fc9da2b9be9ac8d85e

39. Naranbhai V, Kim S, Fletcher H, Cotton MF, Violari A, Mitchell C, et al. The association between the ratio of monocytes:lymphocytes at age 3 months and risk of tuberculosis (TB) in the first two years of life. 2014 Jul 17;12(1):120. Available from: https://search.datacite.org/works/10.1186/s12916014-0120-7.

40. Andrews JR, Nemes E, Tameris M, Landry BS, Mahomed H, McClain JB, et al. Serial QuantiFERON testing and tuberculosis disease risk among young children: an observational cohort study 2017;5:282-90. https://doi.org/10.1016/S2213-2600(17)30060-7.

41. Gupta RK, Turner CT, Venturini C, Esmail H, Rangaka MX, Copas A, et al. Concise whole blood transcriptional signatures for incipient tuberculosis: a systematic review and patient-level pooled meta-analysis 2020;8:395-406. https://doi.org/10.1016/s2213-2600(19)30282-6.

42. Scriba TJ, Fiore-Gartland A, Borate B, Mendelsohn SC, Hadley K, Hikuam C, et al. Biomarker-guided tuberculosis preventive therapy (CORTIS): a randomised controlled trial. 2021 Mar;21(3):354-65. Available from: https://dx.doi.org/10.1016/S1473-3099(20)30914-2.

\section{Tables}

Table 1: Database and keywords

\begin{tabular}{|c|c|c|}
\hline Date & Database & Keywords \\
\hline $\begin{array}{l}25^{\text {th }} \\
\text { May } \\
2020\end{array}$ & PubMed & $\begin{array}{l}\text { (pulmonary tuberculosis[MeSH Terms]) OR (infection, latent } \\
\text { tuberculosis[MeSH Terms])) OR (latent tuberculosis[MeSH Terms])) OR } \\
\text { (mycobacterium tuberculosis[MeSH Terms])) OR ("latent tuberculosis")) OR } \\
(\text { "active tuberculosis")) OR ("active infection")) OR ("latent infection")) OR ("active } \\
\text { tuberculosis infection")) OR ("latent tuberculosis infection")) AND } \\
\text { (biomarkers[MeSH Terms])) OR ("biological markers")) OR ("transcription } \\
\text { biomarker")) OR ("bio signature")) OR (genetic transcription[MeSH Terms])) OR } \\
\text { ("RNA transcription")) OR (proteome[MeSH Terms])) OR } \\
\text { (proteomics[MeSH Terms])) AND (disease progression[MeSH Terms])) OR ("risk } \\
\text { progression")) OR ("attributable risk")) OR ("correlates of risk")) OR ("correlates } \\
\text { of TB risk") }\end{array}$ \\
\hline $\begin{array}{l}25^{\text {th }} \\
\text { May } \\
2020\end{array}$ & HDAS & $\begin{array}{l}\text { ("latent tuberculosis").ti,ab, ("active tuberculosis").ti,ab, ("active infection").ti,a, } \\
\text { ("latent infection").ti,ab, ("latent tuberculosis infection").ti,ab, ("active } \\
\text { tuberculosis infection").ti,ab, ("biological markers").ti,ab, ("transcription } \\
\text { biomarker").ti,ab, ("bio signature").ti,ab, ("pulmonary tuberculosis").ti,ab, ("latent } \\
\text { tuberculosis infection").ti,ab, ("latent tuberculosis").ti,ab, ("active } \\
\text { tuberculosis").ti,ab, ("active tuberculosis infection").ti,ab, ("latent tuberculosis } \\
\text { infection").ti,ab, ("biomarkers").ti,ab, ("transcription biomarker").ti,ab, ("bio } \\
\text { signature").ti,ab, ("risk progression").ti,ab, ("attributable risk").ti,ab, ("correlates of } \\
\text { risk" OR "correlates of TB risk").ti,ab. }\end{array}$ \\
\hline
\end{tabular}

Table 2- Data extraction form 


\begin{tabular}{|l|}
\hline DATA EXTRACTION form \\
\hline First author \\
\hline Year of publication \\
\hline Study title \\
\hline Study setting (country) \\
\hline Study design \\
\hline Sampling strategy \\
\hline Study time period \\
\hline Number of study subjects \\
\hline Number followed-up \\
\hline Age groups of subjects included \\
\hline Characteristics of study group \\
\hline Characteristics of control /comparison group \\
\hline Identification of disease state- latent infection/TB disease \\
\hline Number of subjects who developed TB disease \\
\hline COR type \\
\hline Type of sample used \\
\hline Tests to measure coR \\
\hline Outcomes reported in the study \\
\hline
\end{tabular}

Figures 


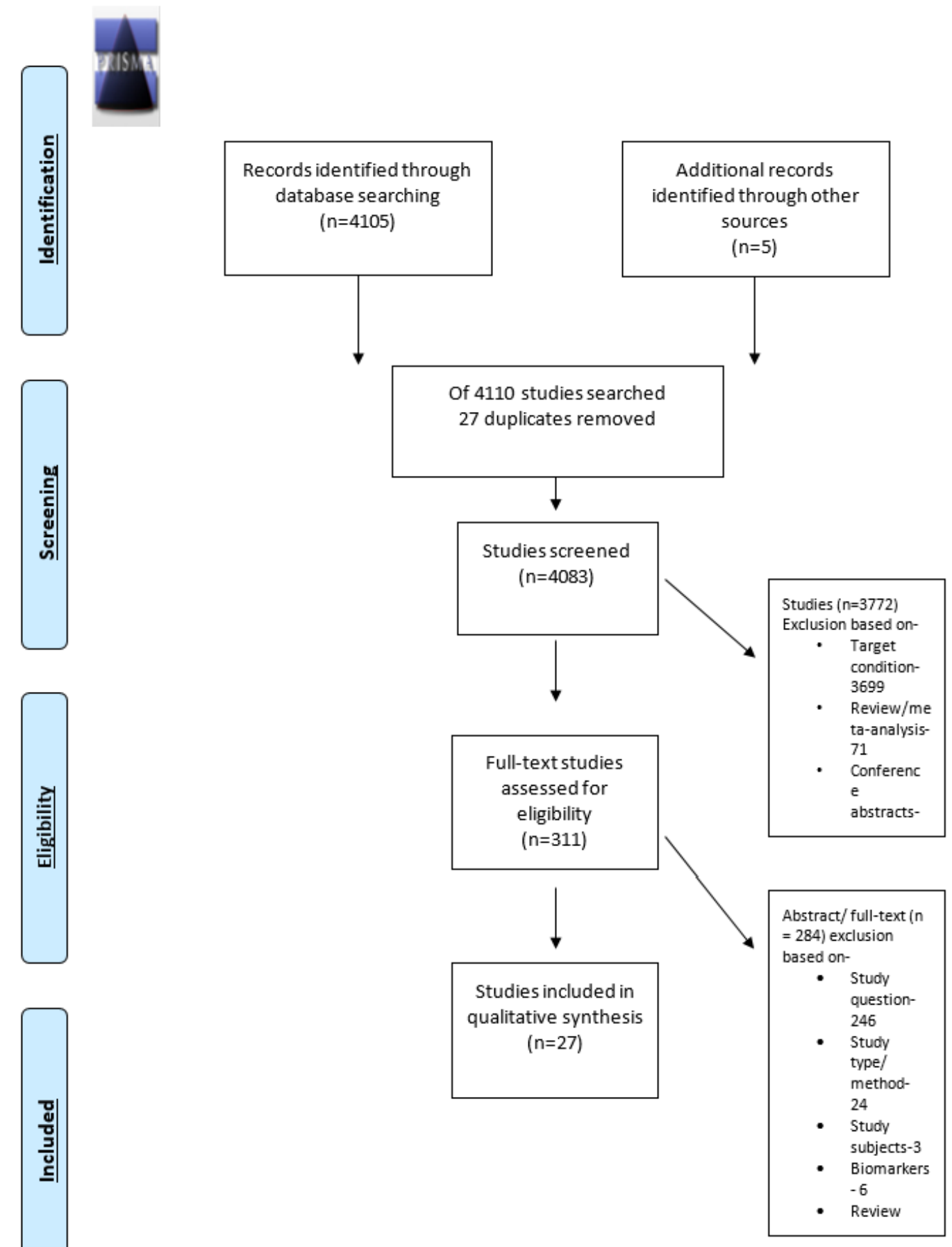

Figure 1

PRISMA 

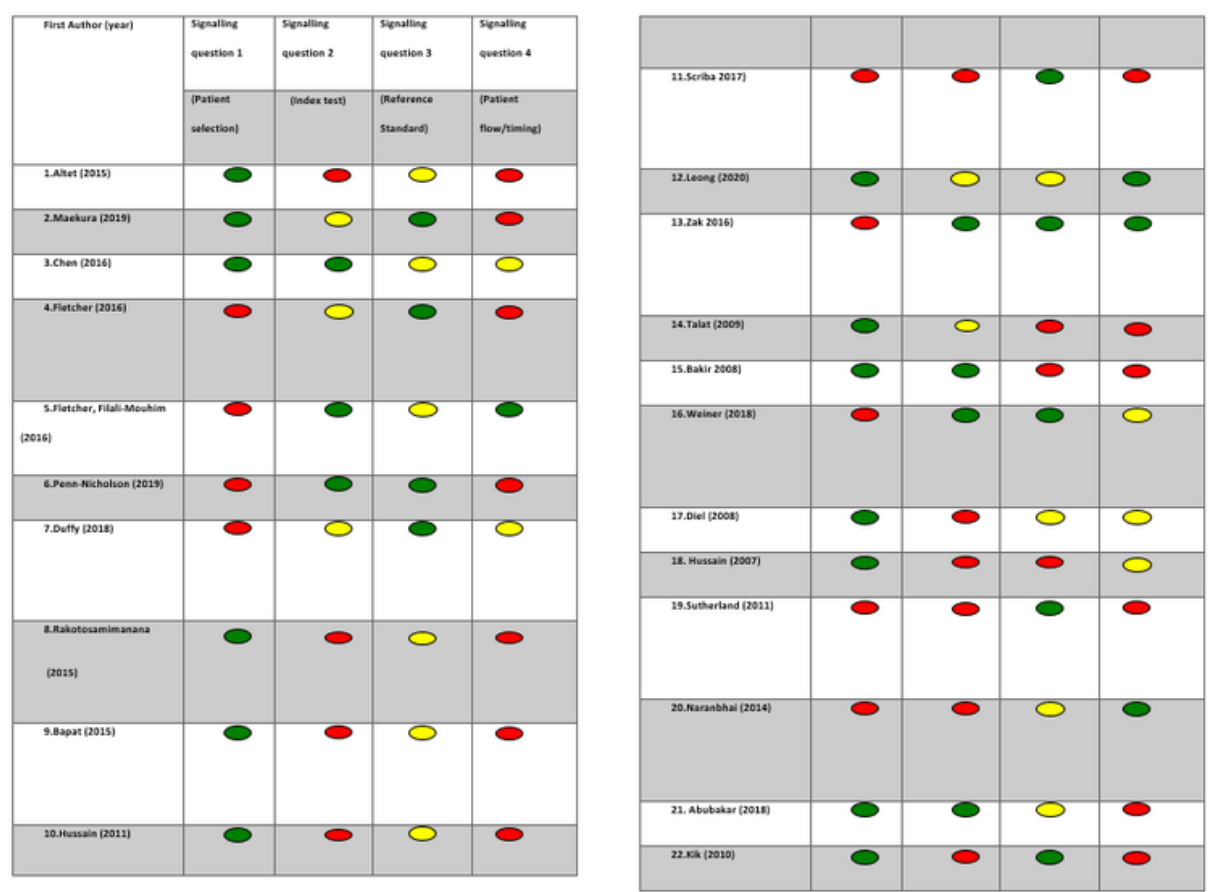

\begin{tabular}{|c|c|c|c|c|}
\hline 21. Andeew (2017) & 0 & 0 & 0 & 0 \\
\hline 24.Mikhalisen [2016) & 0 & 0 & 0 & 0 \\
\hline 25.0elogu (2016) & 0 & 0 & 0 & 8 \\
\hline 26.Warrinke (2018) & 0 & 0 & 0 & 0 \\
\hline 27. Soltiman (2018) & 0 & 0 & 0 & 0 \\
\hline Yes 0 & No & & Unclest & 5 \\
\hline
\end{tabular}

Figure 2

Assessment of Quality of studies (QUADASII)

\section{QUADAS II}

0

$5 \quad 10$

15

20

25

\section{Patient selection \\ Index test \\ Reference standard \\ Patient flow/timing}

\section{Figure 3}

Overall depiction of risk of bias as per QUADAS II 


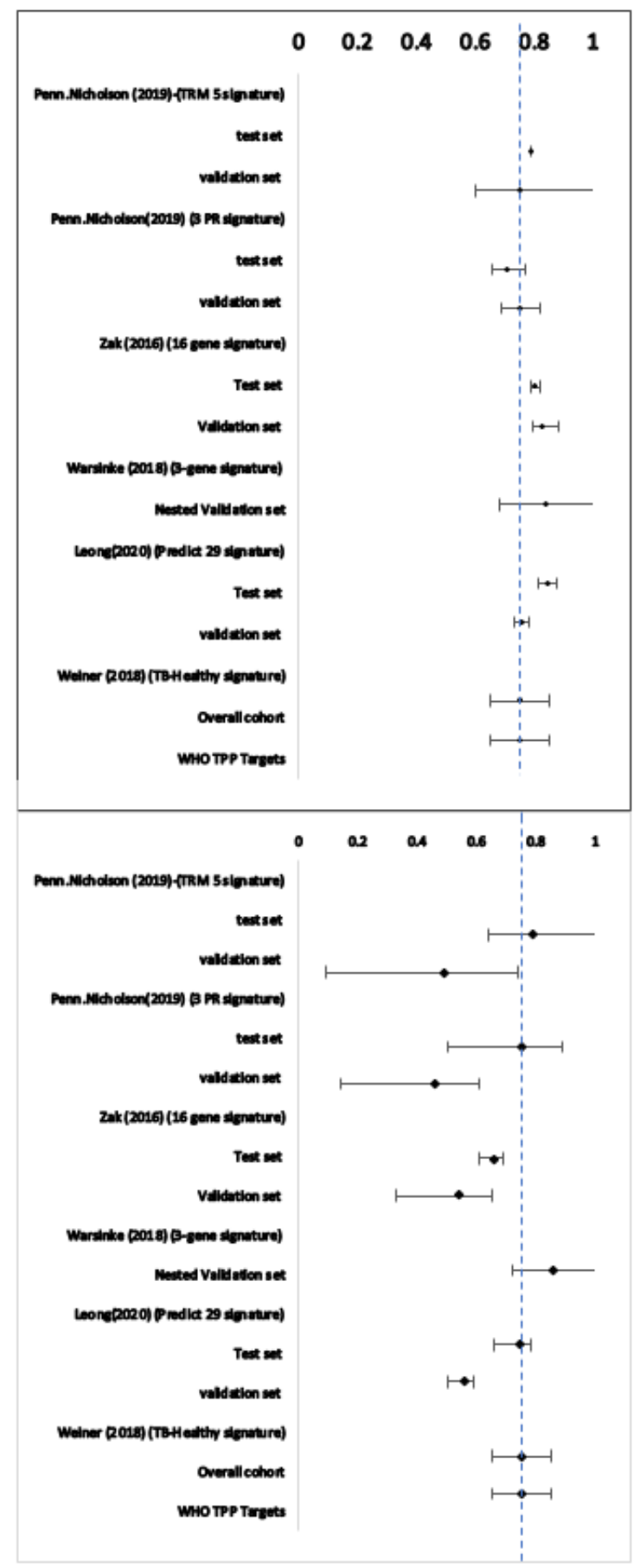

Figure 4

Sensitivity and Specificity for COR compared against WHO TPP Targets

\section{Supplementary Files}

This is a list of supplementary files associated with this preprint. Click to download. 
- supplementaryfileStudiesandoutcomes.docx

- PRISMAAbstractsChecklist.pdf 